\title{
HFSZEEMAN-A program for computing weak and intermediate field fine and hyperfine structure Zeeman splittings from MCDHF wave functions
}

\author{
Martin Andersson ${ }^{\mathrm{a}, *}$, Per Jönsson ${ }^{\mathrm{b}}$ \\ a Department of Physics, Lund University, Box 118, S-221 00 Lund, Sweden \\ ${ }^{\mathrm{b}}$ Nature, Environment, Society, Malmö University, S-205 06 Malmö, Sweden
}

Received 16 August 2006; received in revised form 2 May 2007; accepted 27 July 2007

Available online 8 September 2007

\begin{abstract}
Given electronic wave functions generated by the grasp2K relativistic atomic structure package, this program calculates diagonal magnetic dipole $A_{J}$ and electric quadrupole $B_{J}$ hyperfine interaction constants and Landé $g_{J}$ factors. In addition the program computes diagonal and offdiagonal reduced hyperfine and Zeeman matrix elements and constructs the total interaction matrix for an atom in an external magnetic field. By diagonalizing the interaction matrix and plotting eigenvalues as functions of the magnetic field, Zeeman splittings of hyperfine levels are obtained. The method is applicable in the weak and intermediate field regions and yields results that are useful when analyzing spectra from e.g. EBIT sources and magnetic stars. The program can also be used in the field free limit to calculate mixing coefficients that determine rates of hyperfine induced transitions. For atoms with zero nuclear spin $I$ the program computes splittings of the fine-structure levels.
\end{abstract}

\section{Program summary}

\section{Program title: HFSZEEMAN}

Catalogue identifier: ADZS_v1_0

Program summary URL: http://cpc.cs.qub.ac.uk/summaries/ADZS_v1_0.html

Program obtainable from: CPC Program Library, Queen's University, Belfast, N. Ireland

Licensing provisions: Standard CPC licence, http://cpc.cs.qub.ac.uk/licence/licence.html

No. of lines in distributed program, including test data, etc.: 1528

No. of bytes in distributed program, including test data, etc.: 294664

Distribution format: tar.gz

Programming language: Fortran, Matlab

Computer: IBM-compatible PC, unix workstation

Operating system: Unix, Linux

Classification: 11.6

Subprograms used: Cat Id: ADZL_v1_0; $\quad$ Title: grasp2K v1.0; $\quad$ Reference: CPC 177 (2007) 597

Nature of problem: Prediction of weak and intermediate field Zeeman splittings of fine- and hyperfine structure levels using multiconfiguration Dirac-Hartree-Fock wave functions.

Solution method: The electronic wave function for a state labeled $\Gamma J M$ is expanded in terms of $j j$-coupled configuration state functions $|\Gamma J M\rangle=\sum_{\gamma} c_{\gamma}|\gamma J M\rangle$. In this representation the reduced matrix elements used to construct the interaction matrix can be computed as sums over one-particle radial integrals. By diagonalizing the interaction matrix and plotting eigenvalue as functions of the magnetic field, Zeeman splittings of fine- and hyperfine structure levels are obtained.

Restrictions: The complexity of the cases that can be handled is entirely determined by the grasp2K package [P. Jönsson, H. Xe, C. Froese Fischer, I.P. Grant, Comput. Phys. Commun. 177 (2007) 597] used for the generation of the electronic wave functions.

\footnotetext{
This paper and its associated computer program are available via the Computer Physics Communications homepage on ScienceDirect (http://www.sciencedirect. com/science/journal/00104655).

* Corresponding author.

E-mail address: martin.andersson@fysik.lu.se (M. Andersson).
} 
Running time: CPU time required to execute test cases: a few seconds. (c) 2007 Elsevier B.V. All rights reserved.

PACS: 32.10.Fn; 32.30.-r; 32.60.+i

Keywords: Relativistic atomic wave functions; Hyperfine structure; $A$ and $B$ factors; Landé $g_{J}$ factor; Zeeman effect; Paschen-Back effect; Magnetic field; Multiconfiguration Dirac-Hartree-Fock+Breit

\section{Introduction}

The present hfszeeman program is an extension and update of the old hyperfine structure code hfs92 [1], that was modified by Froese Fischer and Jönsson to treat Landé $g_{J}$ factors [2]. The program is a part of the grasp2K relativistic atomic structure package [3]. As such it adheres to the structure and constraints underlying the package. The role of the program is to calculate magnetic dipole $A_{J}$ and electric quadrupole $B_{J}$ hyperfine interaction constants, Landé $g_{J}$ factors and to determine Zeeman splittings of hyperfine levels in weak and intermediate magnetic fields. For atoms with zero nuclear spin $I$ the program computes Landé $g_{J}$ factors and splittings of the fine-structure levels. Given the output files of the hfszeeman program the accompanying mtrans program [4] can be used to compute transition rates between different magnetic fine- and hyperfine structure sublevels. In the field free limit mtrans can also be used to compute rates of hyperfine induced transitions.

\section{Theory}

\subsection{Relativistic wave functions}

In the multiconfiguration Dirac-Hartree-Fock method the relativistic electronic wave function, frequently referred to as the atomic state function, for a state labeled $\Gamma J M_{J}$ is expanded in $j j$-coupled configuration state functions (CSFs) which are eigenfunctions of $\mathbf{J}^{2}, \mathbf{J}_{z}$ and parity

$$
\left|\Gamma J M_{J}\right\rangle=\sum_{\gamma} c_{\gamma}\left|\gamma J M_{J}\right\rangle
$$

The label $\Gamma$ is often the same as the label for the dominating CSF and gives a short description of the state including the configuration and the coupling tree. The configuration state functions are sums of products of four-component spin-orbitals

$$
\langle\mathbf{r} \mid n \kappa m\rangle=\frac{1}{r}\left(\begin{array}{c}
P_{n \kappa}(r) \chi_{\kappa m}(\hat{r}) \\
i Q_{n \kappa}(r) \chi-\kappa m \\
(\hat{r})
\end{array}\right),
$$

where $n$ is the principal quantum number and $\kappa$ is the relativistic angular quantum number $\kappa= \pm\left(j+\frac{1}{2}\right)$ for $l=j \pm \frac{1}{2}$ with $l$ and $j$ being the orbital and total angular momenta of the electron. $P_{n \kappa}(r)$ and $Q_{n \kappa}(r)$ are the large and small component radial wave functions and $\chi_{\kappa m}(\hat{\mathbf{r}})$ are the spinor spherical harmonics in the $l s j$-coupling scheme

$$
\chi_{\kappa m}(\hat{\mathbf{r}})=\sum_{\sigma}\left\langle l m-\sigma \frac{1}{2} \sigma \mid l \frac{1}{2} j m\right\rangle Y_{l m-\sigma}(\theta, \varphi) \xi(\sigma) .
$$

The radial functions $P_{n \kappa}(r)$ and $Q_{n \kappa}(r)$ are represented on a logarithmic grid and are required to be orthonormal within each $\kappa$ symmetry. In the multiconfiguration self-consistent field procedure both the radial functions and the expansion coefficients for the configuration state functions are optimized to self-consistency.

When the set of radial orbitals has been obtained, relativistic configuration interaction (RCI) calculations can be performed. Here the transverse photon interaction as well as the leading QED effects may be included in the Hamiltonian.

\subsection{Hyperfine structure}

The hyperfine structure of an atomic energy level is caused by the non-central interaction between the electrons and the electromagnetic multipole moments of the nucleus. The Hamiltonian for the interaction may be written as a multipole expansion

$$
H_{h f s}=\sum_{k \geqslant 1} \mathbf{T}^{(k)} \cdot \mathbf{M}^{(k)}
$$

where $\mathbf{T}^{(k)}$ and $\mathbf{M}^{(k)}$ are spherical tensor operators of rank $k$ in the electronic and nuclear spaces, respectively [5]. The $k=1$ term represents the magnetic dipole interaction and the $k=2$ term the electric quadrupole interaction. Higher order terms are smaller 
and can often be neglected. For an $N$-electron atom the electronic tensor operators are, in atomic units,

$$
\begin{aligned}
& \mathbf{T}^{(1)}=\sum_{j=1}^{N} \mathbf{t}^{(1)}(j)=\sum_{j=1}^{N}-i \sqrt{2} \alpha r_{j}^{-2}\left(\boldsymbol{\alpha}_{j} \mathbf{C}^{(1)}(j)\right)^{(1)}, \\
& \mathbf{T}^{(2)}=\sum_{j=1}^{N} \mathbf{t}^{(2)}(j)=\sum_{j=1}^{N}-r_{j}^{-3} \mathbf{C}^{(2)}(j) .
\end{aligned}
$$

In the formulas above, $i=\sqrt{-1}$ is the imaginary unit, $\alpha$ is the fine-structure constant, $\boldsymbol{\alpha}$ is the Dirac matrix and $\mathbf{C}^{(k)}$ is a spherical tensor operator. The matrix elements of the nuclear tensor operators are related to the conventional nuclear magnetic dipole moment $\mu_{I}$ and electric quadrupole moment $Q$ by

$$
\begin{aligned}
& \mu_{I}=\left\langle\Upsilon I I\left|M_{0}^{(1)}\right| \Upsilon I I\right\rangle=\left(\begin{array}{ccc}
I & 1 & I \\
-I & 0 & I
\end{array}\right) \sqrt{2 I+1}\left\langle\Upsilon I\left\|\mathbf{M}^{(1)}\right\| \Upsilon I\right\rangle, \\
& Q=2\left\langle\Upsilon I I\left|M_{0}^{(2)}\right| \Upsilon I I\right\rangle=2\left(\begin{array}{ccc}
I & 2 & I \\
-I & 0 & I
\end{array}\right) \sqrt{2 I+1}\left\langle\Upsilon I\left\|\mathbf{M}^{(2)}\right\| \Upsilon I\right\rangle,
\end{aligned}
$$

where the reduced matrix elements are defined in the Brink and Satchler sense [6]. Evaluating the 3-j symbols we have

$$
\begin{aligned}
& \left\langle\Upsilon I\left\|\mathbf{M}^{(1)}\right\| \Upsilon I\right\rangle=\mu I \sqrt{\frac{I+1}{I}}, \\
& \left\langle\Upsilon I\left\|\mathbf{M}^{(2)}\right\| \Upsilon I\right\rangle=\frac{Q}{2} \sqrt{\frac{(2 I+3)(I+1)}{I(2 I-1)}} .
\end{aligned}
$$

Values of nuclear magnetic dipole and electric quadrupole moments can be found in the recent compilation by Stone [7].

The hyperfine interaction couples the nuclear $\mathbf{I}$ and electronic $\mathbf{J}$ angular momenta to a total momentum $\mathbf{F}=\mathbf{I}+\mathbf{J}$. Denoting the nuclear wave functions by $\left|\Upsilon I M_{I}\right\rangle$, zero-order wave functions of the coupled states can be written

$$
\left|\Upsilon \Gamma I J F M_{F}\right\rangle=\sum_{M_{I}, M_{J}}\left\langle I J M_{I} M_{J} \mid I J F M_{F}\right\rangle\left|\Upsilon I M_{I}\right\rangle\left|\Gamma J M_{J}\right\rangle
$$

If the hyperfine interaction is weak so that the interaction energy is small compared to the fine-structure separation, $H_{h f s}$ can be treated in first-order perturbation theory. A fine-structure level $\Gamma J$ is then split according to

$$
\left\langle\Upsilon \Gamma I J F M_{F}\left|\mathbf{T}^{(1)} \cdot \mathbf{M}^{(1)}+\mathbf{T}^{(2)} \cdot \mathbf{M}^{(2)}\right| \Upsilon \Gamma I J F M_{F}\right\rangle .
$$

We express this in terms of the reduced electronic and nuclear matrix elements

$$
\begin{aligned}
& \left\langle\Upsilon \Gamma I J F M_{F}\left|\mathbf{T}^{(1)} \cdot \mathbf{M}^{(1)}\right| \Upsilon \Gamma I J F M_{F}\right\rangle \\
& =(-1)^{I+J+F}\left\{\begin{array}{lll}
I & J & F \\
J & I & 1
\end{array}\right\} \sqrt{2 J+1} \sqrt{2 I+1}\left\langle\Gamma J\left\|\mathbf{T}^{(1)}\right\| \Gamma J\right\rangle\left\langle\Upsilon I\left\|\mathbf{M}^{(1)}\right\| \Upsilon I\right\rangle, \\
& \langle\Upsilon \\
& \left.\Gamma I J F M_{F}\left|\mathbf{T}^{(2)} \cdot \mathbf{M}^{(2)}\right| \Upsilon \Gamma I J F M_{F}\right\rangle \\
& \quad=(-1)^{I+J+F}\left\{\begin{array}{lll}
I & J & F \\
J & I & 2
\end{array}\right\} \sqrt{2 J+1} \sqrt{2 I+1}\left\langle\Gamma J\left\|\mathbf{T}^{(2)}\right\| \Gamma J\right\rangle\left\langle\Upsilon I\left\|\mathbf{M}^{(2)}\right\| \Upsilon I\right\rangle .
\end{aligned}
$$

Usually the dependence on the $F$ quantum number is factored out and the energies are written in terms of the hyperfine interaction constants

$$
\begin{aligned}
& A_{J}=\frac{\mu_{I}}{I} \frac{1}{\sqrt{J(J+1)}}\left\langle\Gamma J\left\|\mathbf{T}^{(1)}\right\| \Gamma J\right\rangle, \\
& B_{J}=2 Q \sqrt{\frac{J(2 J-1)}{(J+1)(2 J+3)}}\left\langle\Gamma J\left\|\mathbf{T}^{(2)}\right\| \Gamma J\right\rangle .
\end{aligned}
$$

The hyperfine energies are then given by

$$
\frac{1}{2} A_{J} C+B_{J} \frac{\frac{3}{4} C(C+1)-I(I+1) J(J+1)}{2 I(2 I-1) J(2 J-1)},
$$

where $C=F(F+1)-J(J+1)-I(I+1)$. To account for off-diagonal hyperfine effects we consider the total Hamiltonian

$$
H=H_{f s}+H_{h f s}
$$


where $H_{f s}$ is the relativistic fine-structure Hamiltonian that may include the Breit interaction. Now only $F$ and $M_{F}$ are good quantum numbers and we represent the wave function by an expansion

$$
\left|\Upsilon \widetilde{\Gamma} F M_{F}\right\rangle=\sum_{\Gamma J} d_{\Gamma J}\left|\Upsilon \Gamma I J F M_{F}\right\rangle .
$$

This leads to the matrix eigenvalue problem

$$
\mathbf{H d}=E \mathbf{d},
$$

where $\mathbf{H}$ is the matrix with elements

$$
H_{\Gamma J, \Gamma^{\prime} J^{\prime}}=\left\langle\Upsilon \Gamma I J F M_{F}\left|H_{f s}+\mathbf{T}^{(1)} \cdot \mathbf{M}^{(1)}+\mathbf{T}^{(2)} \cdot \mathbf{M}^{(2)}\right| \Upsilon \Gamma^{\prime} I J^{\prime} F M_{F}\right\rangle .
$$

The matrix elements of the fine-structure Hamiltonian $H_{f s}$ are diagonal in all quantum numbers and equals the calculated finestructure energies $E_{\Gamma J}$. The hyperfine interaction matrix elements can be expressed in terms of reduced electronic and nuclear matrix elements. The relevant matrix elements are

$$
\begin{aligned}
& \left\langle\Upsilon \Gamma I J F M_{F}\left|\mathbf{T}^{(1)} \cdot \mathbf{M}^{(1)}\right| \Upsilon \Gamma^{\prime} I J^{\prime} F M_{F}\right\rangle \\
& \quad=(-1)^{I+J+F}\left\{\begin{array}{ccc}
I & J & F \\
J^{\prime} & I & 1
\end{array}\right\} \sqrt{2 J+1} \sqrt{2 I+1}\left\langle\Gamma J\left\|\mathbf{T}^{(1)}\right\| \Gamma^{\prime} J^{\prime}\right\rangle\left\langle\Upsilon I\left\|\mathbf{M}^{(1)}\right\| \Upsilon I\right\rangle,
\end{aligned}
$$

where $J^{\prime}=J-1, J, J+1$ and

$$
\begin{aligned}
& \left\langle\Upsilon \Gamma I J F M_{F}\left|\mathbf{T}^{(2)} \cdot \mathbf{M}^{(2)}\right| \Upsilon \Gamma^{\prime} I J^{\prime} F M_{F}\right\rangle \\
& \quad=(-1)^{I+J+F}\left\{\begin{array}{lll}
I & J & F \\
J^{\prime} & I & 2
\end{array}\right\} \sqrt{2 J+1} \sqrt{2 I+1}\left\langle\Gamma J\left\|\mathbf{T}^{(2)}\right\| \Gamma^{\prime} J^{\prime}\right\rangle\left\langle\Upsilon I\left\|\mathbf{M}^{(2)}\right\| \Upsilon I\right\rangle,
\end{aligned}
$$

where $J^{\prime}=J-2, J-1, J, J+1, J+2$. Explicit formulas for the Wigner 6-j symbols can be found in [8].

\subsection{Zeeman effect of fine-structure levels}

If we choose the direction of the magnetic field as the $z$-direction the interaction, neglecting diamagnetic contributions, can be written

$$
H_{m}=\left(N_{0}^{(1)}+\Delta N_{0}^{(1)}\right) B,
$$

where the last term is the so called Schwinger QED correction. For an $N$-electron atom the electronic tensor operators are [10], in atomic units,

$$
\begin{aligned}
& \mathbf{N}^{(1)}=\sum_{j=1}^{N} \mathbf{n}^{(1)}(j)=\sum_{j=1}^{N}-i \frac{\sqrt{2}}{2 \alpha} r_{j}\left(\boldsymbol{\alpha}_{j} \mathbf{C}^{(1)}(j)\right)^{(1)}, \\
& \Delta \mathbf{N}^{(1)}=\sum_{j=1}^{N} \Delta \mathbf{n}^{(1)}(j)=\sum_{j=1}^{N} \frac{g_{s}-2}{2} \beta_{j} \boldsymbol{\Sigma}_{j},
\end{aligned}
$$

where $\Sigma_{j}$ is the relativistic spin-matrix and $g_{s}=2.00232$ the $g$ factor of the electron spin corrected for QED effects. If the magnetic field is weak so that the interaction energy is small compared to the fine-structure separation the interaction can be treated in firstorder perturbation theory. A fine-structure level $\Gamma J$ is then split according to

$$
\begin{aligned}
& \left\langle\Gamma J M_{J}\left|N_{0}^{(1)}+\Delta N_{0}^{(1)}\right| \Gamma J M_{J}\right\rangle B \\
& \quad=(-1)^{J-M_{J}}\left(\begin{array}{ccc}
J & 1 & J \\
-M_{J} & 0 & M_{J}
\end{array}\right) \sqrt{2 J+1}\left\langle\Gamma J\left\|\mathbf{N}^{(1)}+\Delta \mathbf{N}^{(1)}\right\| \Gamma J\right\rangle B \\
& \quad=\frac{M_{J}}{\sqrt{J(J+1)}}\left\langle\Gamma J\left\|\mathbf{N}^{(1)}+\Delta \mathbf{N}^{(1)}\right\| \Gamma J\right\rangle B .
\end{aligned}
$$

Usually the dependence on the $M_{J}$ quantum number is factored out and the energies are expressed in terms of the Lande $g_{J}$ factor

$$
g_{J}=2 \frac{\left\langle\Gamma J\left\|\mathbf{N}^{(1)}+\Delta \mathbf{N}^{(1)}\right\| \Gamma J\right\rangle}{\sqrt{J(J+1)}} .
$$

The energy splittings are then given by

$$
g_{J} M_{J} \frac{B}{2}
$$


In the intermediate field regime we consider the total Hamiltonian

$$
H=H_{f s}+H_{m} .
$$

When the field is included only $M_{J}$ remain a good quantum number and the wave function is written as an expansion

$$
\left|\widetilde{\Gamma} M_{J}\right\rangle=\sum_{\Gamma J} d_{\Gamma J}\left|\Gamma J M_{J}\right\rangle .
$$

Just as for the hyperfine interaction this leads to a matrix eigenvalue problem

$$
\mathbf{H d}=E \mathbf{d},
$$

where $\mathbf{H}$ is the matrix with elements

$$
H_{\Gamma J, \Gamma^{\prime} J^{\prime}}=\left\langle\Gamma J M_{J}\left|H_{f s}+\left(N_{0}^{(1)}+\Delta N_{0}^{(1)}\right) B\right| \Gamma^{\prime} J^{\prime} M_{J}\right\rangle .
$$

The matrix elements of the fine-structure Hamiltonian $H_{f s}$ are diagonal in all quantum numbers and equals the energies $E_{\Gamma J}$ of the fine-structure levels. The Zeeman interaction matrix elements can be expressed in terms of the reduced electronic matrix elements and the magnetic field

$$
\begin{aligned}
& \left\langle\Gamma M_{J}\left|N_{0}^{(1)}+\Delta N_{0}^{(1)}\right| \Gamma^{\prime} J M_{J}\right\rangle \\
& \quad=(-1)^{J-M_{J}}\left(\begin{array}{ccc}
J & 1 & J \\
-M_{J} & 0 & M_{J}
\end{array}\right) \sqrt{2 J+1}\left\langle\Gamma J\left\|\mathbf{N}^{(1)}+\Delta \mathbf{N}^{(1)}\right\| \Gamma^{\prime} J\right\rangle \\
& \quad=\frac{M_{J}}{\sqrt{J(J+1)}}\left\langle\Gamma J\left\|\mathbf{N}^{(1)}+\Delta \mathbf{N}^{(1)}\right\| \Gamma^{\prime} J\right\rangle, \\
& \left\langle\Gamma J M_{J}\left|N_{0}^{(1)}+\Delta N_{0}^{(1)}\right| \Gamma^{\prime} J-1 M_{J}\right\rangle \\
& \quad=(-1)^{J-M_{J}}\left(\begin{array}{ccc}
J & 1 & J-1 \\
-M_{J} & 0 & M_{J}
\end{array}\right) \sqrt{2 J+1}\left\langle\Gamma J\left\|\mathbf{N}^{(1)}+\Delta \mathbf{N}^{(1)}\right\| \Gamma^{\prime} J-1\right\rangle \\
& \quad=\sqrt{\frac{J^{2}-M_{J}^{2}}{J(2 J-1)}\left\langle\Gamma J\left\|\mathbf{N}^{(1)}+\Delta \mathbf{N}^{(1)}\right\| \Gamma^{\prime} J-1\right\rangle .}
\end{aligned}
$$

By diagonalizing the interaction matrix and plotting eigenvalues as functions of the magnetic field Zeeman splittings of finestructure levels are obtained.

\subsection{Zeeman effect of hyperfine levels}

We now go on to describe the Zeeman effect of hyperfine levels. If we choose the direction of the magnetic field as the $z$-direction the interaction, again neglecting diamagnetic contributions, can be written

$$
H_{m}=\left(N_{0}^{(1)}+\Delta N_{0}^{(1)}\right) B+\text { interaction with nucleus. }
$$

The interaction with the nucleus is weak and can be neglected. Below we will treat both the weak field limit as well as the intermediate field case.

If the magnetic field is weak so that the interaction energy is small compared to the hyperfine structure separation the interaction can be treated in first-order perturbation theory. A hyperfine level $\Upsilon \Gamma I J F$ for which off-diagonal effects are small is then split according to

$$
\begin{aligned}
\langle\Upsilon & \left.\Gamma I J F M_{F}\left|N_{0}^{(1)}+\Delta N_{0}^{(1)}\right| \Upsilon \Gamma I J F M_{F}\right\rangle B \\
& =M_{F} \frac{F(F+1)+J(J+1)-I(I+1)}{2 F(F+1)} \frac{\left\langle\Gamma J\left\|\mathbf{N}^{(1)}+\Delta \mathbf{N}^{(1)}\right\| \Gamma J\right\rangle}{\sqrt{J(J+1)}} B \\
& =M_{F} \frac{F(F+1)+J(J+1)-I(I+1)}{2 F(F+1)} g_{J} \frac{B}{2} .
\end{aligned}
$$

By defining a Landé $g_{F}$ factor

$$
g_{F}=\frac{F(F+1)+J(J+1)-I(I+1)}{2 F(F+1)} g_{J},
$$

the energy splittings are given by

$$
g_{F} M_{F} \frac{B}{2}
$$

in analogy with the formula for the splitting of the fine-structure levels. 
More generally we will look at hyperfine structure, including off-diagonal contributions, in an intermediate field. The total Hamiltonian is now given by

$$
H=H_{f s}+H_{h f s}+H_{m} .
$$

In this case $M_{F}$ is the only good quantum number and we represent the wave function by the expansion

$$
\left|\Upsilon \tilde{\Gamma} I M_{F}\right\rangle=\sum_{\Gamma J F} d_{\Gamma J F}\left|\Upsilon \Gamma I J F M_{F}\right\rangle .
$$

This leads to the matrix eigenvalue problem

$$
\mathbf{H d}=E \mathbf{d},
$$

where $\mathbf{H}$ is the matrix with elements

$$
H_{\Gamma J F, \Gamma^{\prime} J^{\prime} F^{\prime}}=\left\langle\Upsilon \Gamma I J F M_{F}\left|H_{f s}+\mathbf{T}^{(1)} \cdot \mathbf{M}^{(1)}+\mathbf{T}^{(2)} \cdot \mathbf{M}^{(2)}+\left(N_{0}^{(1)}+\Delta N_{0}^{(1)}\right) B\right| \Upsilon \Gamma^{\prime} I J^{\prime} F^{\prime} M_{F}\right\rangle .
$$

The matrix elements of the hyperfine operator are diagonal in $F$ and have been treated in a previous section. The Zeeman matrix elements between the coupled atomic and nuclear wave functions are given by

$$
\begin{aligned}
& \left\langle\Upsilon \Gamma I J F M_{F}\left|N_{0}^{(1)}+\Delta N_{0}^{(1)}\right| \Upsilon \Gamma^{\prime} I J^{\prime} F M_{F}\right\rangle \\
& \quad=M_{F} \sqrt{\frac{2 F+1}{F(F+1)}}(-1)^{I+J^{\prime}+1+F}\left\{\begin{array}{ccc}
J & F & I \\
F & J^{\prime} & 1
\end{array}\right\} \sqrt{2 J+1}\left\langle\Gamma J\left\|\mathbf{N}^{(1)}+\Delta \mathbf{N}^{(1)}\right\| \Gamma^{\prime} J^{\prime}\right\rangle,
\end{aligned}
$$

where $J^{\prime}=J-1, J, J+1$ and

$$
\begin{aligned}
& \left\langle\Upsilon \Gamma I J F M_{F}\left|N_{0}^{(1)}+\Delta N_{0}^{(1)}\right| \Upsilon \Gamma^{\prime} I J^{\prime} F-1 M_{F}\right\rangle \\
& \quad=\sqrt{\frac{F^{2}-M_{F}^{2}}{F}(-1)^{I+J^{\prime}+1+F}\left\{\begin{array}{ccc}
J & F & I \\
F-1 & J^{\prime} & 1
\end{array}\right\} \sqrt{2 J+1}\left\langle\Gamma J\left\|\mathbf{N}^{(1)}+\Delta \mathbf{N}^{(1)}\right\| \Gamma^{\prime} J^{\prime}\right\rangle,}
\end{aligned}
$$

where $J^{\prime}=J-1, J, J+1$.

\subsection{Computation of reduced matrix elements}

In the multiconfiguration approximation

$$
\left|\Gamma J M_{J}\right\rangle=\sum_{\gamma} c_{\gamma}\left|\gamma J M_{J}\right\rangle
$$

a general electronic matrix element can be written

$$
\left\langle\Gamma J\left\|\mathbf{W}^{(k)}\right\| \Gamma^{\prime} J^{\prime}\right\rangle=\sum_{\gamma, \gamma^{\prime}} c_{\gamma} c_{\gamma^{\prime}}\left\langle\gamma J\left\|\mathbf{W}^{(k)}\right\| \gamma^{\prime} J^{\prime}\right\rangle .
$$

The evaluation of the electronic matrix element between configuration state functions is done using a program originally written by Pyper, Grant and Beatham, [9] where angular recoupling programs are set up to reduce the matrix element $\left\langle\gamma J\left\|\mathbf{W}^{(k)}\right\| \gamma^{\prime} J^{\prime}\right\rangle$ to terms that involve single-particle orbitals only

$$
\left\langle\gamma J\left\|\mathbf{W}^{(k)}\right\| \gamma^{\prime} J^{\prime}\right\rangle=\sum_{a, b} d_{a b}^{k}\left\langle n_{a} \kappa_{a}\left\|\mathbf{w}^{(k)}\right\| n_{b} \kappa_{b}\right\rangle .
$$

The single-particle matrix elements $\left\langle n_{a} \kappa_{a}\left\|\mathbf{w}^{(k)}\right\| n_{b} \kappa_{b}\right\rangle$, in turn, can be factorized into a reduced angular matrix elements and radial integrals. Here we give the factorization of the single-particle matrix elements corresponding to the electronic magnetic dipole and electric quadrupole hyperfine operators and to the magnetic operators [10]

$$
\begin{aligned}
& \left\langle n_{a} \kappa_{a}\left\|\mathbf{t}^{(1)}\right\| n_{b} \kappa_{b}\right\rangle=-\alpha\left(\kappa_{a}+\kappa_{b}\right)\left\langle-\kappa_{a}\left\|\mathbf{C}^{(1)}\right\| \kappa_{b}\right\rangle\left[r^{-2}\right]_{n_{a} \kappa_{a} n_{b} \kappa_{b}}, \\
& \left\langle n_{a} \kappa_{a}\left\|\mathbf{t}^{(2)}\right\| n_{b} \kappa_{b}\right\rangle=-\left\langle\kappa_{a}\left\|\mathbf{C}^{(2)}\right\| \kappa_{b}\right\rangle\left\langle r^{-3}\right\rangle_{n_{a} \kappa_{a} n_{b} \kappa_{b}}, \\
& \left\langle n_{a} \kappa_{a}\left\|\mathbf{n}^{(1)}\right\| n_{b} \kappa_{b}\right\rangle=-\frac{1}{2 \alpha}\left\langle-\kappa_{a}\left\|\mathbf{C}^{(1)}\right\| \kappa_{b}\right\rangle[r]_{n_{a} \kappa_{a} n_{b} \kappa_{b}}, \\
& \left\langle n_{a} \kappa_{a}\left\|\Delta \mathbf{n}^{(1)}\right\| n_{b} \kappa_{b}\right\rangle=\frac{g_{s}-2}{2}\left(\kappa_{a}+\kappa_{b}-1\right)\left\langle-\kappa_{a}\left\|\mathbf{C}^{(1)}\right\| \kappa_{b}\right\rangle\left\langle r^{0}\right\rangle_{n_{a} \kappa_{a} n_{b} \kappa_{b}},
\end{aligned}
$$


where

$$
\begin{aligned}
\left\langle\kappa\left\|\mathbf{C}^{(k)}\right\| \kappa^{\prime}\right\rangle & =(-1)^{j^{\prime}-k-1 / 2} \sqrt{2 j^{\prime}+1}\left(\begin{array}{ccc}
j & j^{\prime} & k \\
\frac{1}{2} & -\frac{1}{2} & 0
\end{array}\right) \pi\left(l, k, l^{\prime}\right), \\
\pi\left(l, k, l^{\prime}\right) & = \begin{cases}1 & \text { if } l+k+l^{\prime} \text { even }, \\
0 & \text { otherwise }\end{cases}
\end{aligned}
$$

and

$$
\begin{aligned}
{\left[r^{k}\right]_{n \kappa n^{\prime} \kappa^{\prime}} } & =\int_{0}^{\infty} r^{k}\left[P_{n \kappa}(r) Q_{n^{\prime} \kappa^{\prime}}(r)+Q_{n \kappa}(r) P_{n^{\prime} \kappa^{\prime}}(r)\right] d r, \\
\left\langle r^{k}\right\rangle_{n \kappa n^{\prime} \kappa^{\prime}} & =\int_{0}^{\infty} r^{k}\left[P_{n \kappa}(r) P_{n^{\prime} \kappa^{\prime}}(r)+Q_{n \kappa}(r) Q_{n^{\prime} \kappa^{\prime}}(r)\right] d r .
\end{aligned}
$$

\section{Installation of programs}

The hyperfine Zeeman package consists of a Fortran program hfszeeman and a Matlab ${ }^{1}$ program plothfszeeman that allows the computed energy structure to be plotted. Installation of the programs assumes that the grasp2K package is available and that a number of environment variables have been set as described in [3].

Start the installation procedure by copying the hfszeeman. tar.gz file to the grasp $2 \mathrm{~K} / \mathrm{src} / \mathrm{app}$ / v2 directory. Uncompress the tar file and give the unix tar xvf command. This will build the Fortran source code under the directory hfszeeman. The Matlab source code is in the subdirectory hfszeeman/matlab and data files needed to run the test cases appear in the subdirectories hfszeeman/data_Ge and hfszeeman/data_He.

Once the environment variables are properly set up, the making of the hfszeeman executable is straightforward. Simply change to the $\mathrm{hfszeeman}$ directory and issue the make command. If the compilation and linking is successful the executable will automatically be transfered to the grasp $2 \mathrm{~K} / \mathrm{bin}$ directory. Changing to the data directories, test runs described later in the article can now be executed to make sure the hfs zeeman program works correctly.

Matlab is a interpreting language and there is no need for compilation for this part. To make sure that the plothfszeeman program is working start Matlab and add the hfszeeman/matlab directory to the path. Change to the data directories and execute the test runs.

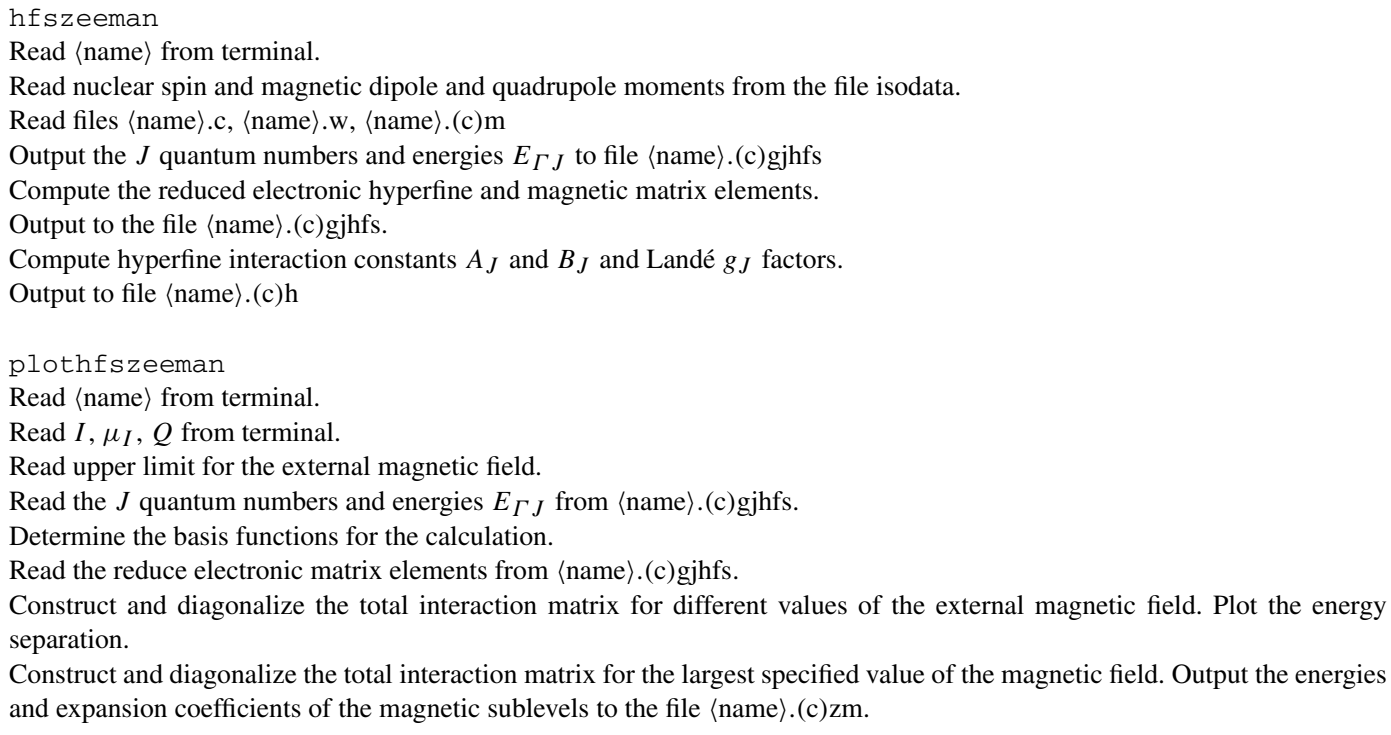

Fig. 1. Program operation and data flow for hfszeeman and plothfszeeman.

\footnotetext{
${ }^{1}$ Matlab is a registered trademark of The MathWorks, Inc.
} 


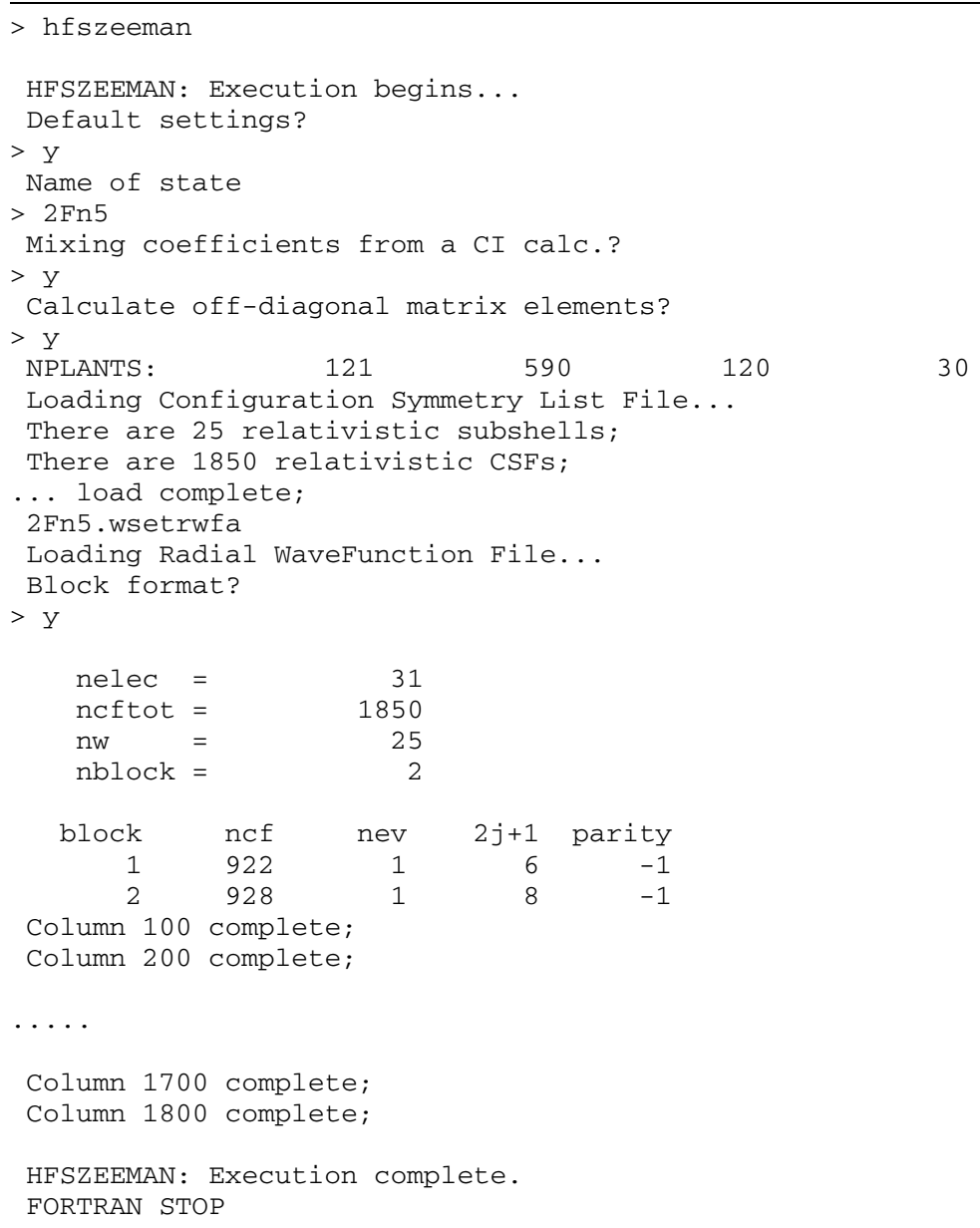

Fig. 2. Session log for the hfszeeman program. The nuclear spin and magnetic dipole and quadrupole moments are read from the file isodata.

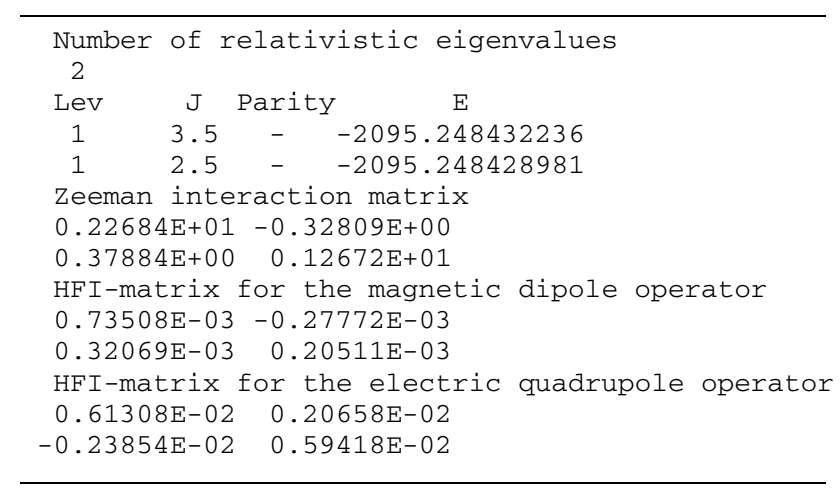

Fig. 3. The hfszeeman output file $2 \mathrm{Fn} 5 . \mathrm{cgjhfs}$ containing $J$ quantum numbers and energies for the fine-structure levels $4 s^{2} 4 f^{2} F_{5} / 2,7 / 2$ together with reduced electronic matrix elements of the magnetic interaction.

\section{Program structure}

\subsection{HFSZEEMAN}

hfszeeman computes and outputs the Landé $g_{J}$ factors for all states $\Gamma J M_{J}$ in a multiconfiguration Dirac-Hartree-Fock (MCDHF) or relativistic configuration interaction (RCI) calculation. If the nuclear spin, $I$, is non-zero hfs zeeman also computes and outputs the hyperfine interaction constants $A_{J}$ and $B_{J}$. In addition the program computes and outputs the reduced electronic hyperfine and magnetic matrix elements between the states in three different matrices. To the output from the hfs $z$ eeman program, $J$ quantum numbers and energies $E_{\Gamma J}$ of all the fine-structure states from the preceding MCDHF or RCI calculations are added. 


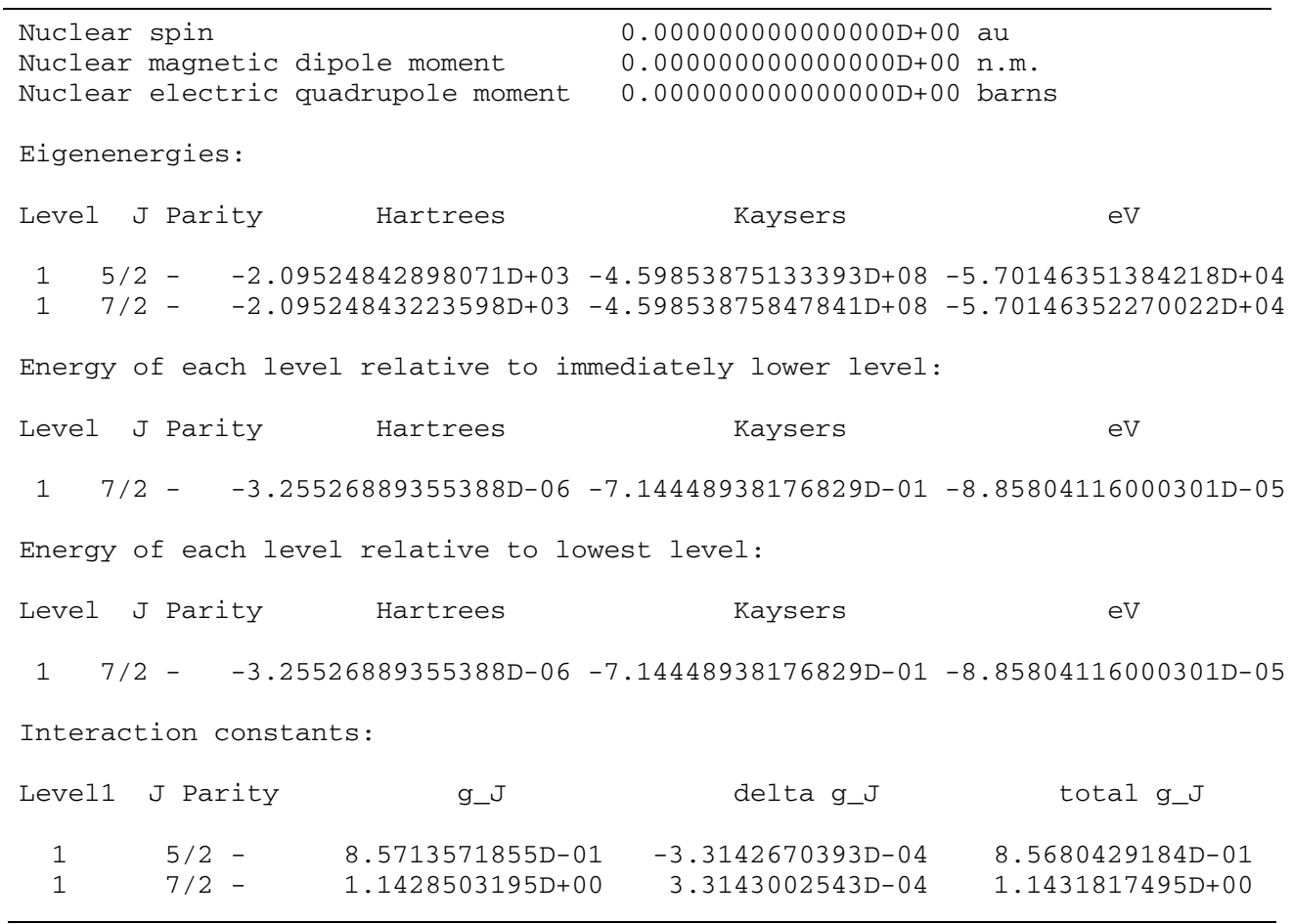

Fig. 4. The hfszeeman output file $2 \mathrm{Fn} 5$. ch with nuclear data, $J$ quantum numbers, energies and Landé $g_{J}$ factors the for fine-structure levels $4 s^{2} 4 f^{2} F_{5 / 2}, 7 / 2$.

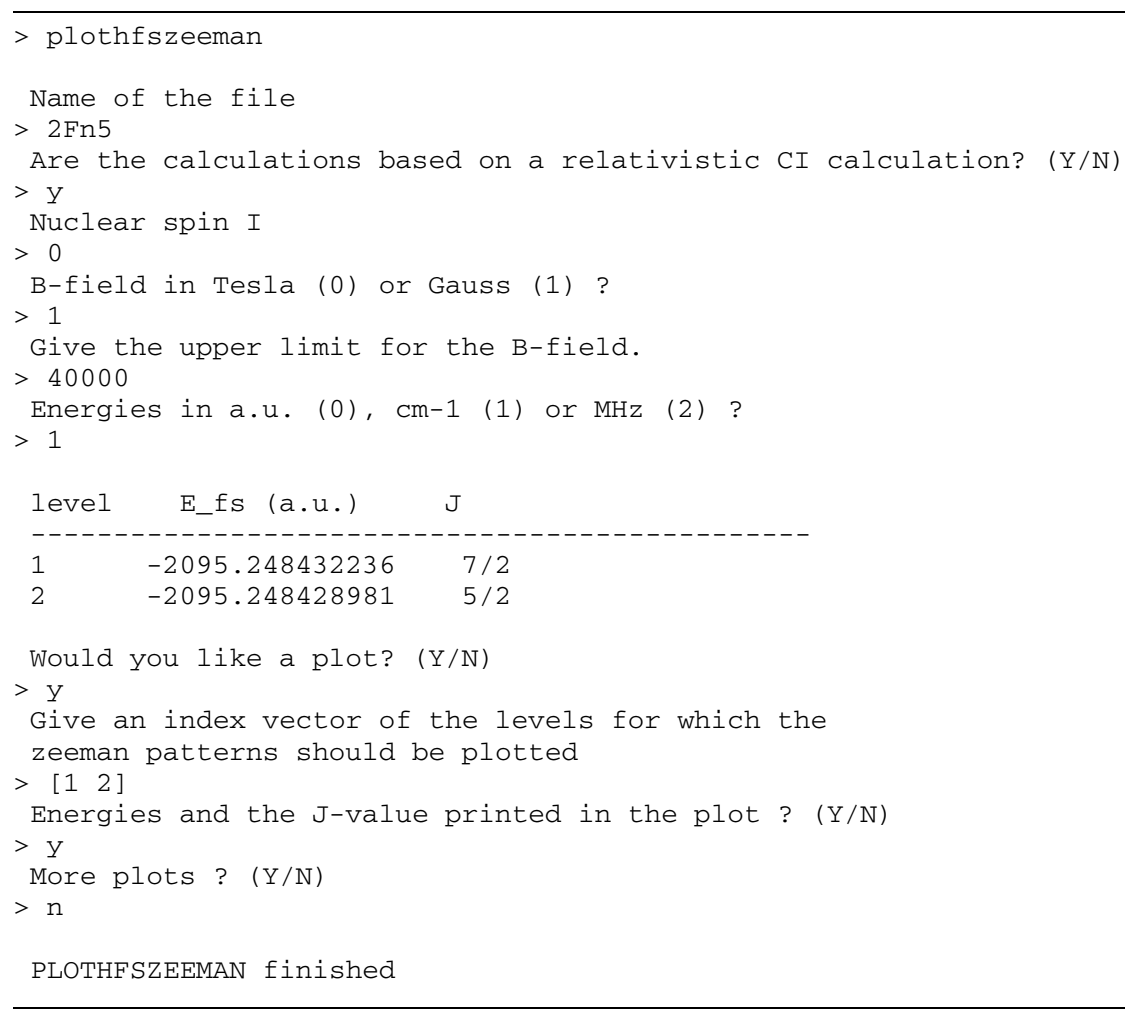

Fig. 5. Session log for the plothf szeeman program. Magnetic sublevels are plotted for $4 s^{2} 4 f^{2} F_{5 / 2,7 / 2}$.

\subsection{PLOTHFSZEEMAN}

plothfszeeman reads the nuclear spin $I$, the nuclear magnetic dipole moment $\mu_{I}$, the nuclear electric quadrupole moment $Q$ and the maximum magnetic field $B$ from the terminal and the reduced matrix elements from the hfszeeman output file. From the list of atomic states and the nuclear spin $I$ the program determines the basis functions for the calculation. If the nuclear spin $I$ 


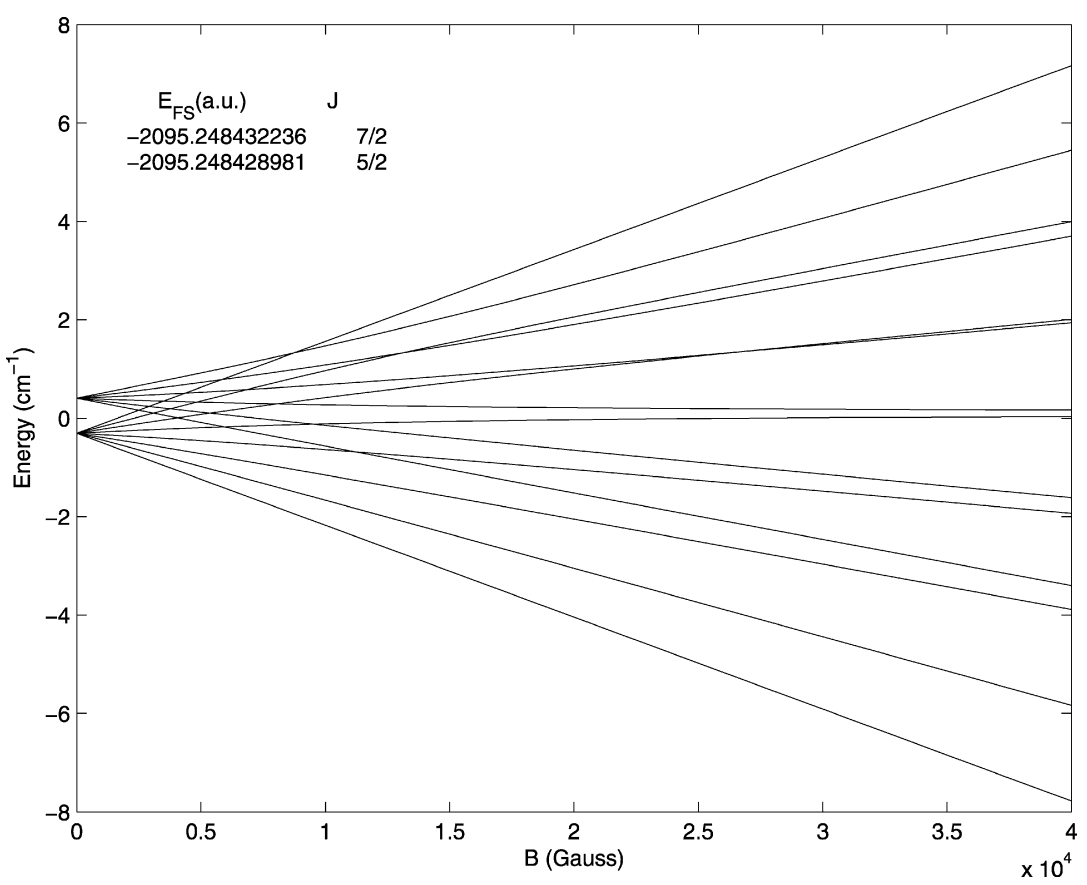

Fig. 6. Magnetic sublevels of $4 s^{2} 4 f^{2} F_{5 / 2,7 / 2}$ as functions of the magnetic field. The displayed energy levels are relative to the weighted energy average at zero field.

is non-zero the basis functions are

$$
\left|\Upsilon \Gamma I J F M_{F}\right\rangle=\sum_{M_{I}, M_{J}}\left\langle I J M_{I} M_{J} \mid I J F M_{F}\right\rangle\left|\Upsilon I M_{I}\right\rangle\left|\Gamma J M_{J}\right\rangle
$$

with $|I-J| \leqslant F \leqslant I+J$. In cases where the nuclear spin $I$ is zero the basis functions are instead given by the atomic state functions $\left|\Gamma J M_{J}\right\rangle$. The program goes on and constructs and diagonalizes the interaction matrix for different values $B$ of the magnetic field and plots the energies of the magnetic sublevels as functions of $B$. For the largest specified value of the magnetic field the energies of the magnetic sublevels and the expansion coefficients $d_{\Gamma J F}$ (or $d_{\Gamma J}$ when the nuclear spin $I$ is zero) for the basis functions are written to file.

The calculations in plothfszeeman are normally done in double precision arithmetic. In some case it may, however, be necessary to switch to variable precision arithmetics which gives more digits.

The operation and data flow (see next section) of hfszeeman and plothfszeeman is schematically shown in Fig. 1.

\section{Input and output data}

\subsection{HFSZEEMAN}

This program assumes the naming convention of the grasp $2 \mathrm{~K}$ package and the $\langle$ name $\rangle$ of the state has to be specified. The program reads data from the configuration list file $\langle$ name $\rangle . c$, the wave function file $\langle$ name $\rangle$.w and, dependent on whether the electronic wave functions originate from a MCDHF or RCI calculation, from the mixing file $\langle$ name $\rangle . \mathrm{m}$ or $\langle$ name $\rangle$.cm. Values of the nuclear spin, magnetic dipole and quadrupole moments are, just as for all programs of the grasp $2 \mathrm{~K}$ package, read from the file isodata.

The program produces two output files $\langle$ name $\rangle$.(c)gjhfs and $\langle$ name $\rangle$.(c)h, where the letter c in the extension indicates that the input data were from an RCI calculation. In the file $\langle$ name $\rangle$.(c)gjhfs the program outputs the $J$ quantum numbers and energies $E_{\Gamma} J$ of the atomic states together with the reduced matrix elements. Note that the reduced matrix elements are not symmetric but related according to (Brink and Satchler [6, p. 61])

$$
\sqrt{2 J+1}\left\langle J\left\|\mathbf{T}^{(k)}\right\| J^{\prime}\right\rangle=(-1)^{J-J^{\prime}} \sqrt{2 J^{\prime}+1}\left\langle J^{\prime}\left\|\mathbf{T}^{(k)}\right\| J\right\rangle
$$

In the file $\langle$ name $\rangle$.(c)h the program outputs the energies and the diagonal Landé $g_{J}$ factors, and if the isotope has a nuclear spin, the diagonal $A_{J}$ and $B_{J}$ hyperfine constants. 


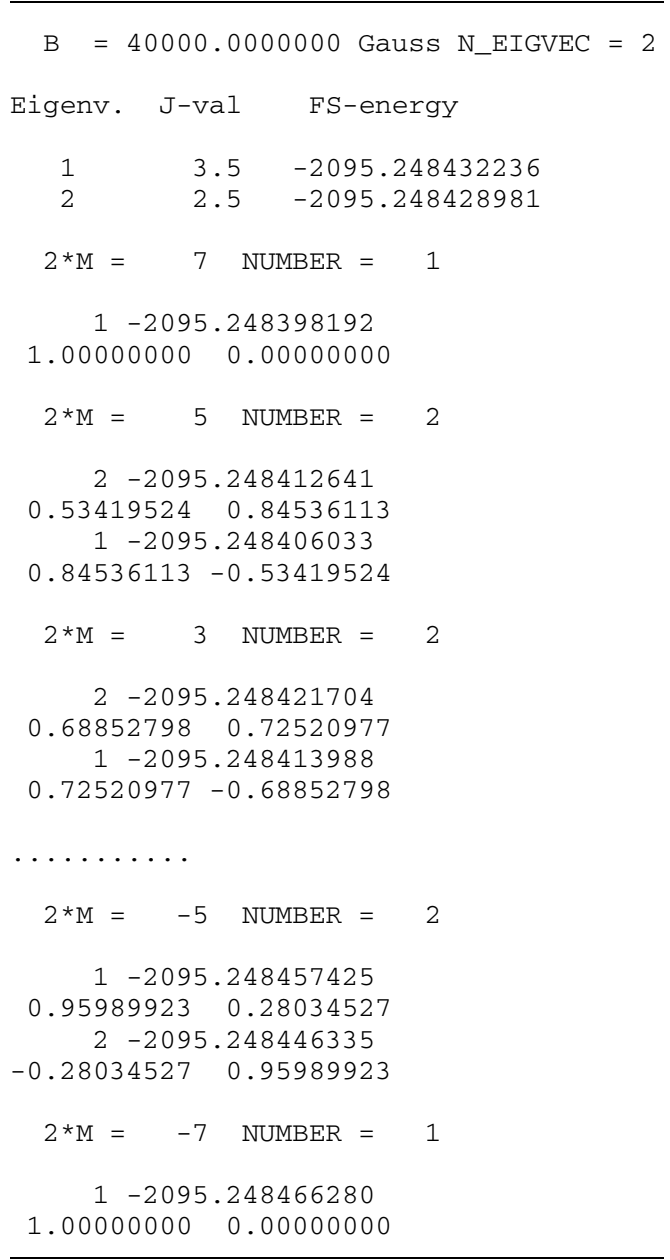

Fig. 7. The file $2 \mathrm{Fn} 5$. c zm with energies and mixing coefficients of the magnetic sublevels of the fine-structure levels $4 s^{2} 4 f^{2} F_{5 / 2,7 / 2}$ at a magnetic field of 40000 Gauss.

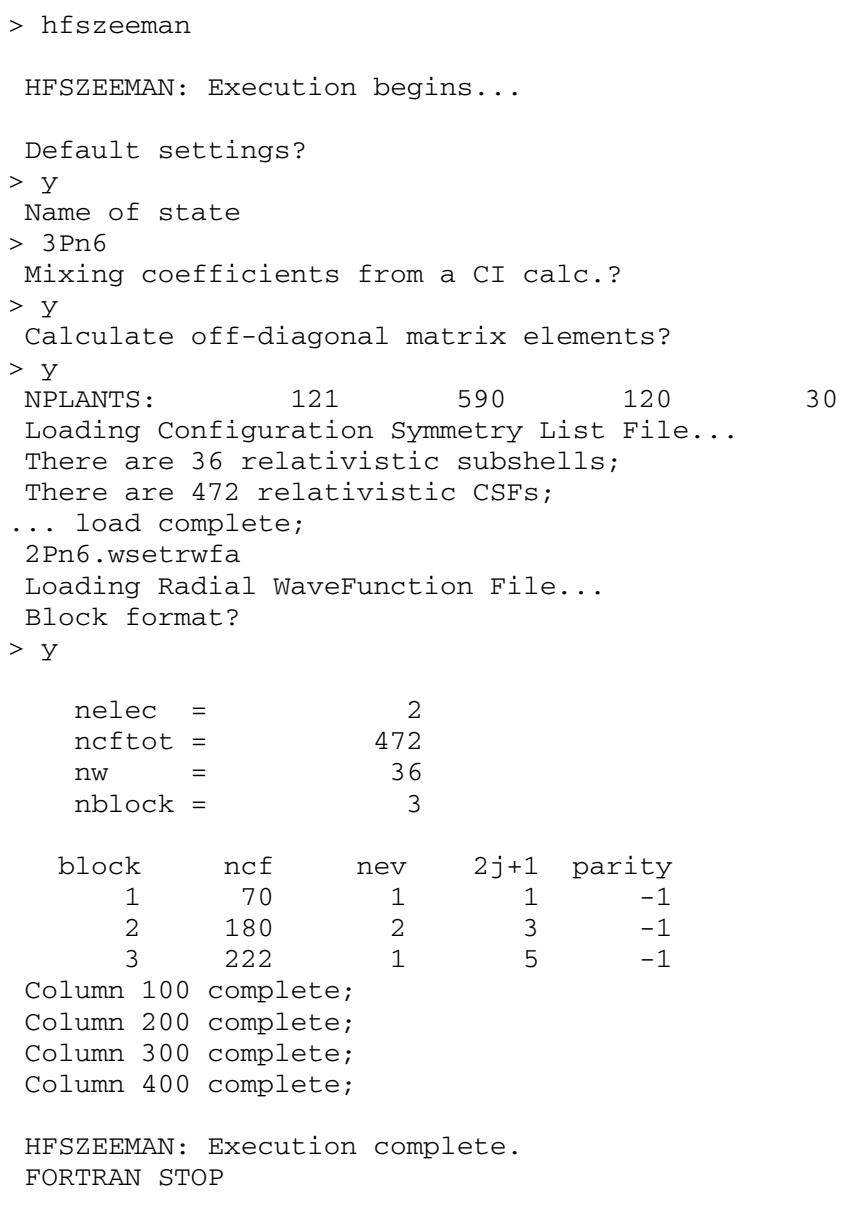

Fig. 8. Session log for the hf s zeeman program. The nuclear spin and magnetic dipole and quadrupole moments are read from the file isodata.

\subsection{PLOTHFSZEEMAN}

Given the $\langle$ name $\rangle$ the program opens and reads $\langle$ name $\rangle$.(c)gjhfs. The program asks for the nuclear spin. If the spin is given a nonzero value, values for the nuclear magnetic dipole and electric quadrupole moments need to be supplied. The maximum magnetic field should be given, in Tesla or Gauss, and the units of energy should be chosen to a.u., $\mathrm{cm}^{-1}$ or MHz. The program plots the energy of the magnetic sublevels as a function of the magnetic field for fine- or hyperfine levels selected by the user. The field free energies together with some further information about the fine/hyperfine levels are printed to the file $\langle$ name $\rangle$.(c)zm, together with the energies and expansion coefficients of the magnetic sublevels for the largest specified value of the magnetic field.

\section{Examples}

In the test run output we present two different calculations; the first is for an isotope in Ge without nuclear spin where the splittings of two fine-structure levels are plotted as functions of the magnetic field, the second is for ${ }^{3} \mathrm{He}$ which has a nuclear spin, $I=1 / 2$, and here the splittings of a number of hyperfine levels are displayed. This test run was compared to [11], and Fig. 12 was found to be in excellent agreement with the similar plot in [11].

To test the programs additional calculations, not presented in the test run output, have been done. The diagonal and off-diagonal reduced electronic magnetic matrix elements in light atoms were checked against the corresponding elements in pure $L S$-coupling [12]. A calculation was done to determine the splitting of the two hyperfine levels of $1 s^{2} 2 s^{2} 2 p^{6} 3 s^{2} S_{1 / 2}$ in Sodium and the result was in perfect agreement with the Breit-Rabi formula [13]. Calculations were also done in the field free limit for the hyperfine levels of $1 s 2 p^{3} P$ in Helium-like Beryllium for comparison with experiment [14] and other theoretical calculations [15], and a detailed agreement was found. Similar calculations were also made for the hyperfine levels derived from the same fine-structure levels as above for Helium-like Fluorine. Here the off-diagonal hyperfine interaction elements are large compared to the fine-structure 


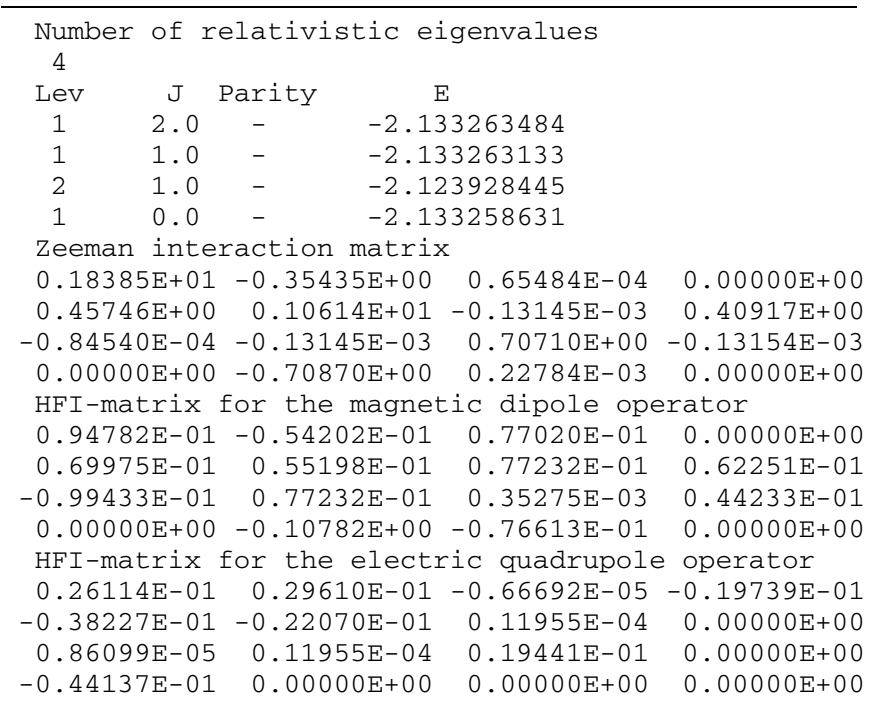

Fig. 9. The hfszeeman output file $3 \mathrm{Pn} 6 . \operatorname{cgjhfs}$ containing $J$ quantum numbers and energies for the fine-structure levels of $1 s 2 p^{1,3} P$ together with reduced electronic matrix elements of the hyperfine and magnetic interaction.

\begin{tabular}{|c|c|c|c|}
\hline \multirow{3}{*}{$\begin{array}{l}\text { Nuclear } \\
\text { Nuclear } \\
\text { Nuclear }\end{array}$} & spin & \multicolumn{2}{|c|}{$5.000000000000000 \mathrm{D}-01 \mathrm{au}$} \\
\hline & magnetic dipole moment & $2.127497720000000 \mathrm{D}+00$ & $0 \mathrm{n} . \mathrm{m}$. \\
\hline & electric quadrupole moment & $0.000000000000000 \mathrm{D}+00$ & 0 barns \\
\hline \multicolumn{4}{|c|}{ Eigenenergies: } \\
\hline Level J & Hartrees & Kaysers & eV \\
\hline 1 & $-2.13325863106008 D+00$ & $-4.68196150196834 D+05-$ & $-5.80489458068455 D+01$ \\
\hline 1 & $-2.13326313263103 D+00$ & $-4.68197138177454 \mathrm{D}+05$ & $-5.80490683008760 D+01$ \\
\hline 2 & $-2.12392844472579 D+00$ & $-4.66148410996939 D+05-$ & $-5.77950584098832 \mathrm{D}+01$ \\
\hline 1 & $-2.13326348364110 \mathrm{D}+00$ & $-4.68197215215261 D+05-$ & $-5.80490778523503 D+01$ \\
\hline
\end{tabular}

Energy of each level relative to immediately lower level:

\begin{tabular}{lrrrrr} 
Level & \multicolumn{2}{l}{ J Parity } & Hartrees & \multicolumn{1}{c}{ Kaysers } & eV \\
1 & 1 & - & $-4.50157094356030 \mathrm{D}-06$ & $-9.87980620317715 \mathrm{D}-01$ & $-1.22494030467627 \mathrm{D}-04$ \\
2 & 1 & - & $9.33468790523939 \mathrm{D}-03$ & $2.04872718051548 \mathrm{D}+03$ & $2.54009890992817 \mathrm{D}-01$ \\
1 & 2 & - & $-9.33503891531640 \mathrm{D}-03$ & $-2.04880421832249 \mathrm{D}+03$ & $-2.54019442467093 \mathrm{D}-01$
\end{tabular}

Energy of each level relative to lowest level:

$$
\begin{aligned}
& \text { Level J Parity Hartrees Kaysers eV } \\
& 1 \quad 1-\quad-4.50157094356030 \mathrm{D}-06-9.87980620317715 \mathrm{D}-01-1.22494030467627 \mathrm{D}-04 \\
& 2 \quad 1-9.33018633429583 \mathrm{D}-03 \quad 2.04773919989516 \mathrm{D}+03 \quad 2.53887396962350 \mathrm{D}-01 \\
& 12-\quad-4.85258102056463 \mathrm{D}-06-1.06501842733320 \mathrm{D}+00-1.32045504743184 \mathrm{D}-04
\end{aligned}
$$

Interaction constants:

$\begin{array}{cccccccc}\text { Level1 J } & \text { Parity } & \text { A }(\mathrm{MHz}) & \text { B }(\mathrm{MHz}) & \text { 9_J } & \text { delta g_J } \\ 1 & 1 & - & 2.1713872786 \mathrm{D}+03 & 0.0000000000 \mathrm{D}+00 & 1.4999565728 \mathrm{D}+00 & 1.1599965276 \mathrm{D}-03 & 1.5011165693 \mathrm{D}+00 \\ 2 & 1 & - & 1.3876531139 \mathrm{D}+01 & 0.000000000 \mathrm{D}+00 & 9.9999359428 \mathrm{D}-01 & 2.5473373189 \mathrm{D}-09 & 9.9999359682 \mathrm{D}-01 \\ 1 & 2 & - & 2.1526837767 \mathrm{D}+03 & 0.0000000000 \mathrm{D}+00 & 1.4999582945 \mathrm{D}+00 & 1.1599999886 \mathrm{D}-03 & 1.5011182945 \mathrm{D}+00\end{array}$

Fig. 10. The hfszeeman output file 3 Pn 6 . ch with nuclear data, $J$ quantum numbers, energies and Landé $g_{J}$ factors for the fine-structure levels of $1 s 2 p^{1,3} P$. 


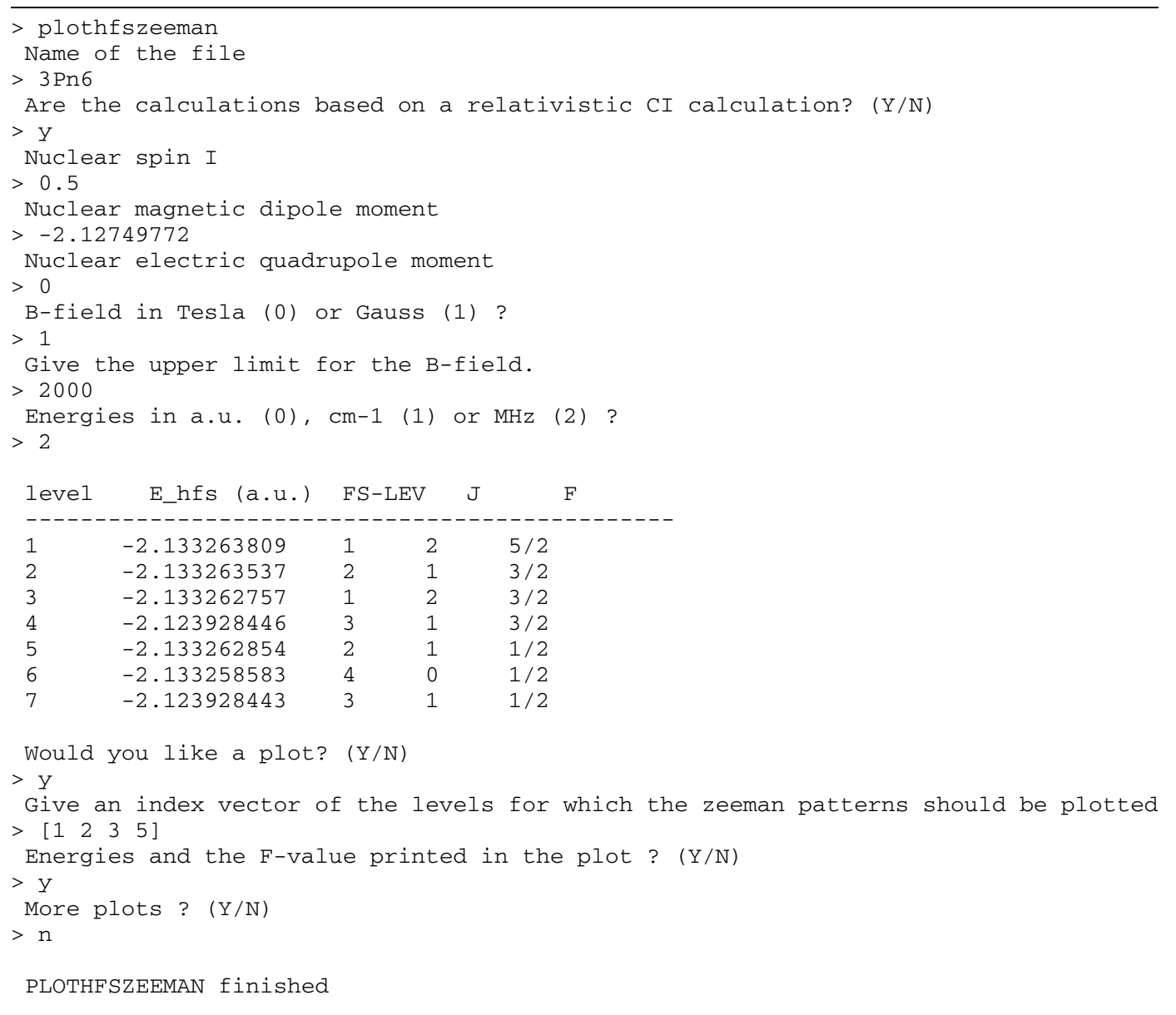

Fig. 11. Session log for the plothfs zeeman program. Magnetic sublevels are plotted for the hyperfine levels $1 s 2 p^{3} P_{2}, F=5 / 2,3 / 2$ and $1 s 2 p^{3} P_{1}, F=3 / 2,1 / 2$ in ${ }^{3} \mathrm{He}$. FS-LEV is used to identify which hyperfine levels that are derived from the same fine-structure level.

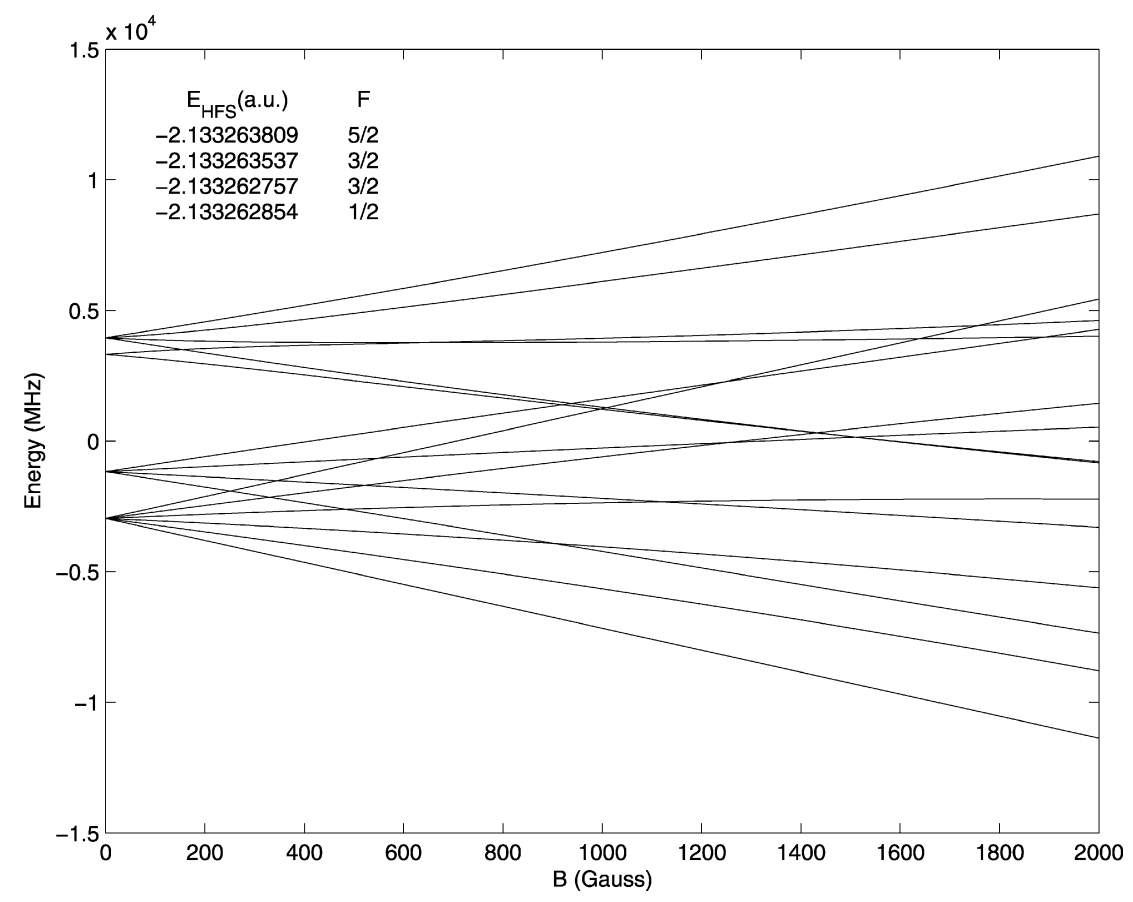

Fig. 12. Magnetic sublevels of the hyperfine levels of $1 s 2 p^{3} P_{1,2}$ as functions of the magnetic field. The displayed energy levels are relative to the weighted average energy at zero field. 


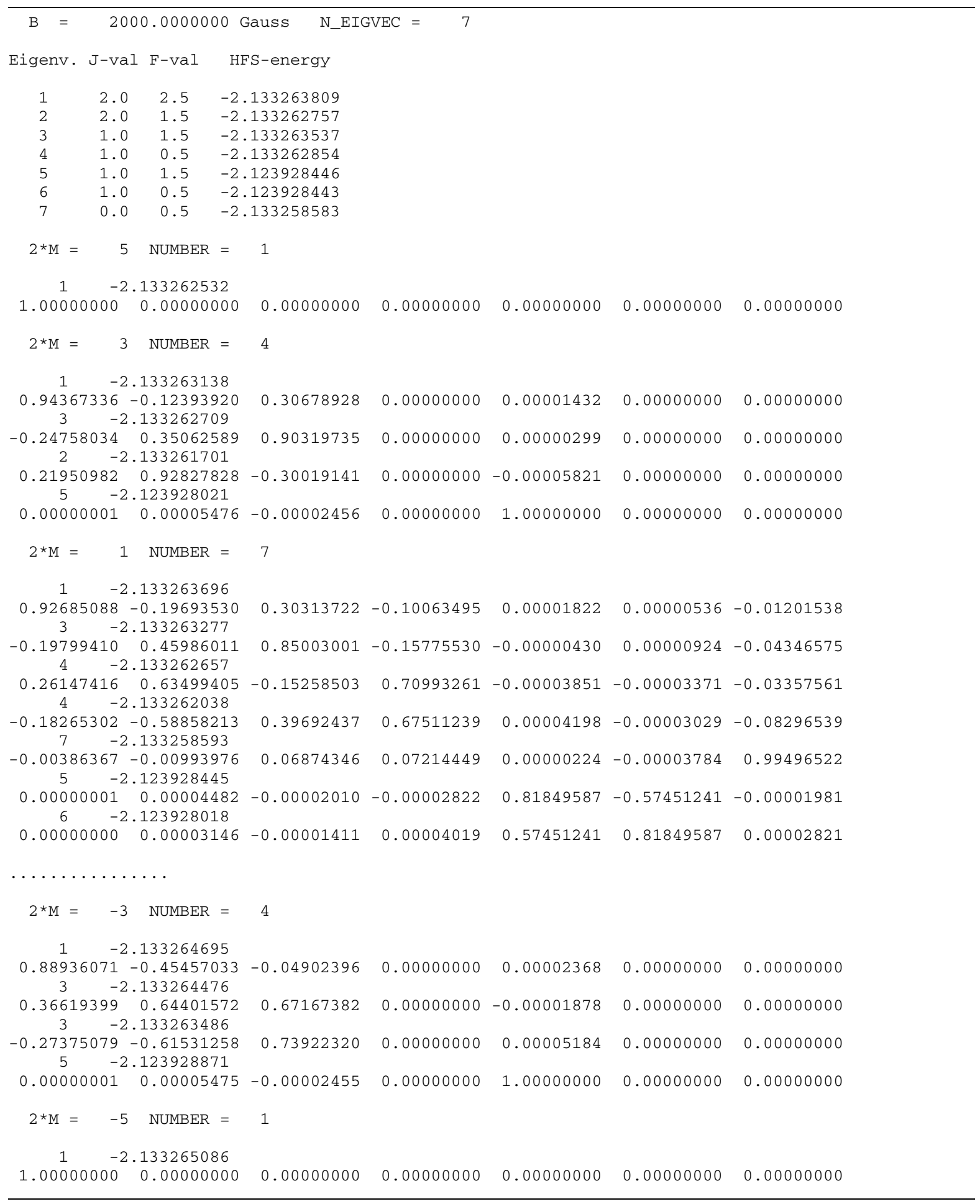

Fig. 13. The file 3 Pn $6 . \mathrm{zm}$ with energies and mixing coefficients of the magnetic sublevels of the hyperfine levels of $1 s 2 p^{3} P_{0,1,2}$ and $1 s 2 p^{1} P_{1}$ in ${ }^{3} \mathrm{He}$ at a magnetic field of 2000 Gauss.

splittings and have a large impact on the energies. No experimental values are available, but again there is an excellent agreement with [15]. As a further test, the programs were applied to the $4 s 4 d^{3} D_{2}-4 s 4 f^{3} F_{2,3}$ transitions in Ga II, where the off-diagonal hyperfine interaction is necessary to explain the features of recent Fourier transform spectra [16].

\section{Acknowledgement}

This work was supported by the Swedish Research Council (Vetenskapsrådet). 


\section{Appendix A. Test run output}

The first test run is for the two fine-structure levels $4 s^{2} 4 f^{2} F_{5 / 2,7 / 2}$ in Ge II. The test run generates Landé $g_{J}$ factors for each of the two levels together with energies and mixing coefficients of the magnetic sublevels for the largest value of the given magnetic field. In addition the energies of the magnetic sublevels of $4 s^{2} 4 f^{2} F_{5 / 2,7 / 2}$ are plotted as functions of the magnetic field. Some preparatory calculations using the programs of grasp $2 \mathrm{~K}$ have to be done in order to perform the test run. First, lists of configuration state functions (CSFs) are generated using $j$ jgen. Then the radial wave functions are estimated using erwf, and by using mcp2 and rscf2 multiconfiguration Dirac-Hartree-Fock calculations are done. In our test case the Breit interaction and leading QED effects are included in subsequent relativistic configuration interaction calculations using $r \mathrm{ci} 2$. The calculations account for correlation between the three outer electrons and includes orbitals with principal quantum numbers up to $n=5$. The largest configuration expansion consists of $1850 \mathrm{CSFs}$ and is in the file named $2 \mathrm{Fn} 5$. c. The radial wave functions are in $2 \mathrm{Fn} 5 . \mathrm{w}$ and the mixing coefficients from rci2 are saved in $2 \mathrm{Fn} 5$. c. These files reside in the grasp $2 \mathrm{~K} / \mathrm{src} / \mathrm{appl} / \mathrm{v} 2 / \mathrm{hfszeeman/data \_ Ge} \mathrm{directory.}$ We can now run the hfszeeman program, and the session log is displayed in Fig. 2. hfszeeman produces two output files; 2Fn5.cgjhfs (see Fig. 3) which gives the reduced electronic hyperfine and magnetic interaction matrices and $2 \mathrm{Pn} 5$. ch (see Fig. 4) which displays the Landé $g_{J}$ factors. Using $2 \mathrm{Fn} 5 . \mathrm{cgjhfs}$ we can run plothfszeeman, see Fig. 5, to produce the plot in Fig. 6 and the mixing file 2Fn5. czm, see Fig. 7.

The next test run is for the hyperfine levels of $1 s 2 p^{1,3} P$ in neutral ${ }^{3} \mathrm{He}$. This isotope has a nuclear spin of $1 / 2$, a nuclear dipole moment of $-2.12749772 \mathrm{n} . \mathrm{m}$. The test run produces Landé $g_{J}$ factors for the fine-structure levels, $A_{J}$ and $B_{J}$ hyperfine interaction constants and mixing coefficients for the magnetic hyperfine sublevels at the magnetic field 2000 Gauss. In addition a plot of the energies of the magnetic sublevels of $1 s 2 p^{3} P_{1,2}$ as functions of the magnetic field is produced. The plot is compared with a similar plot by $\mathrm{Wu}$ and Drake [11].

The preparatory work follows the steps outlined above and we add a large number of correlation orbitals in an accurate calculation. The generated configuration list $3 \mathrm{Pn} 6 . \mathrm{c}$, the radial wave functions $3 \mathrm{Pn} 6 . \mathrm{w}$ and the mixing file $3 \mathrm{Pn} 6 . \mathrm{cm}$ can be found in grasp2K/src/appl/v2/hfszeeman/data_He. We run hfszeeman and the session log is depicted in Fig. 8. The program produces two files; $3 \mathrm{Pn} 6 . \mathrm{cgjhfs}$ which contains the reduced electronic hyperfine and magnetic interaction matrices (see Fig. 9) and $3 \mathrm{Pn} 6$. ch, see Fig. 10, which shows the Landé $g_{J}$ factor and the hyperfine interaction constants $A_{J}$ and $B_{J}$ for each of the fine-structure levels. Using $3 \mathrm{Pn} 6 . \mathrm{cgjhfs}$ we run plothfs zeeman, see Fig. 11, to produce a plot of the energies of the $1 s 2 p^{3} P_{1,2}$ hyperfine levels as functions of the magnetic field, Fig. 12, and a file $3 \mathrm{Pn} 6$. c $\mathrm{m}$ with the magnetic hyperfine sublevels and mixing coefficients at the magnetic field of 2000 Tesla, Fig. 13.

\section{References}

[1] P. Jönsson, F.A. Parpia, C. Froese Fischer, Comput. Phys. Commun. 96 (1996) 301.

[2] C. Froese Fischer, P. Jönsson, J. Mol. Struct. 537 (2001) 55.

[3] P. Jönsson, X. He, C. Froese Fischer, I.P. Grant, Comput. Phys. Commun. 177 (2007) 597.

[4] M. Andersson, P. Jönsson, Comput. Phys. Commun. (2007), in preparation.

[5] I. Lindgren, A. Rosén, Case Stud. At. Phys. 4 (1974) 97.

[6] D.M. Brink, G.R. Satchler, Angular Momentum, Clarendon Press, Oxford, 1993.

[7] N.J. Stone, At. Data Nucl. Data Tables 90 (2005) 75.

[8] I.I. Sobelman, Atomic Spectra and Radiative Transitions, Springer-Verlag, Berlin, Heidelberg, New York, 1979.

[9] N.C. Pyper, I.P. Grant, N. Beatham, Comput. Phys. Commun. 15 (1978) 387.

[10] K.T. Cheng, W.J. Childs, Phys. Rev. A 31 (1985) 2775.

[11] Q. Wu, G.W.F. Drake, J. Phys. B: At. Mol. Opt. Phys. 40 (2007) 393.

[12] P. Jönsson, S. Gustafsson, Comput. Phys. Commun. 144 (2002) 188.

[13] G. Breit, I.I. Rabi, Phys. Rev. 38 (1931) 2082.

[14] T.J. Scholl, R. Cameron, S.D. Rosner, L. Zhang, R.A. Holt, C.J. Sansonetti, J.D. Gillaspy, Phys. Rev. Lett. 71 (1993) 2188

[15] W.R. Johnson, K.T. Cheng, D.R. Plante, Phys. Rev. A 55 (1997) 2728.

[16] M. Andersson, P. Jönsson, J. Phys. B 39 (2006) 4239. 